\title{
XMM-NEWTON SLEW SURVEY OBSERVATIONS OF THE GRAVITATIONAL WAVE EVENT GW150914
}

\author{
E. Troja ${ }^{1,2}$, A. M. Read ${ }^{3}$, A. Tiengo ${ }^{4,5}$, and R. Salvaterra ${ }^{5}$ \\ ${ }^{1}$ NASA Goddard Space Flight Center, 8800 Greenbelt Rd, Greenbelt, MD 20771, USA \\ 2 Department of Physics and Astronomy, University of Maryland, College Park, MD 20742-4111, USA \\ ${ }^{3}$ Department of Physics and Astronomy, Leicester University, Leicester LE1 7RH, UK \\ ${ }^{4}$ Istituto Universitario di Studi Superiori, piazza della Vittoria 15, I-27100 Pavia, Italy \\ ${ }^{5}$ Istituto di Astrofisica Spaziale e Fisica Cosmica Milano, INAF, via E. Bassini 15, I-20133 Milano, Italy \\ Received 2016 March 21; accepted 2016 March 29; published 2016 April 22
}

\begin{abstract}
The detection of the first gravitational wave (GW) transient GW150914 prompted an extensive campaign of follow-up observations at all wavelengths. Although no dedicated XMM-Newton observations have been performed, the satellite passed through the GW150914 error region during normal operations. Here we report the analysis of the data taken during these satellite slews performed two hours and two weeks after the GW event. Our data cover 1.1 and $4.8 \mathrm{deg}^{2}$ of the final GW localization region. No X-ray counterpart to GW150914 is found down to a sensitivity of $6 \times 10^{-13} \mathrm{erg} \mathrm{cm}^{-2} \mathrm{~s}^{-1}$ in the $0.2-2 \mathrm{keV}$ band. Nevertheless, these observations show the great potential of XMM-Newton slew observations for searching for the electromagnetic counterparts of GW events. A series of adjacent slews performed in response to a GW trigger would take $\lesssim 1.5$ days to cover most of the typical GW credible region. We discuss this scenario and its prospects for detecting the X-ray counterpart of future GW detections.
\end{abstract}

Key words: gamma-ray burst: general - gravitational waves - X-rays: general

\section{INTRODUCTION}

On 2015 September 14 at 09:50:45 UTC, the advanced Laser Interferometer Gravitational-Wave Observatory (LIGO; Aasi et al. 2015) triggered and detected its first gravitational wave (GW) event, dubbed GW150914 (Abbott et al. 2016b). This was a revolutionary discovery, which confirmed the predictions of general relativity (Abbott et al. 2016f) and opened a new window into the study of our universe. The refined LIGO analysis yields a robust detection (Abbott et al. 2016c), and shows that the observed waveform is consistent with the merger of a binary black hole (BBH) system with relatively "heavy" masses of $\approx 36 M_{\odot}$ and $\approx 29 M_{\odot}$, thus providing the first observational evidence that these astrophysical systems exist (Belczynski et al. 2010; Abbott et al. 2016a).

Despite its crude localization $\left(\approx 590 \mathrm{deg}^{2}\right)$, GW150914 enjoyed an extensive observing campaign across the electromagnetic spectrum (Abbott et al. 2016e). Although no visible counterpart was expected from a BBH merger, Connaughton et al. (2016) reported the presence of a weak, short duration $(\sim 1 \mathrm{~s})$ gamma-ray excess, starting $0.4 \mathrm{~s}$ after the $\mathrm{GW}$ trigger and coming from a sky area consistent with the GW localization. This event was not detected during the more sensitive INTEGRAL observations (Savchenko et al. 2016), thus its nature, as well as its association with GW150914, remain uncertain. Nonetheless, several new scenarios were proposed in order to explore the possible electromagnetic signatures of a BBH merger, including short gamma-ray bursts and their accompanying afterglows (e.g., Murase et al. 2016; Perna et al. 2016). In this respect, X-ray observations of GW events represent a promising route toward the discovery of their electromagnetic counterparts. The X-ray window offers several advantages when compared to other energy bands: (a) most $(\sim 80 \%)$ short gamma-ray bursts (GRBs) have an X-ray afterglow detection (D'Avanzo et al. 2014), compared to only $30 \%$ detected in the optical band (Kann et al. 2011), showing that X-ray observations are far more efficient in detecting these events; (b) space-based X-ray observations are not subject to atmospheric constraints, which often hamper or degrade ground-based searches; and (c) the number of X-ray candidates expected within the LIGO localizations is significantly lower than in deep optical surveys.

The major obstacle for X-ray observatories is to rapidly observe the large GW error regions with an adequate sensitivity. In the case of GW1509014, the entire LIGO error region was scanned by MAXI with a shallow sensitivity of $\approx 10^{-9} \mathrm{erg} \mathrm{cm}^{-2} \mathrm{~s}^{-1}$ in the $2-20 \mathrm{keV}$ energy band (Abbott et al. 2016e). Deeper Target of Opportunity (ToO) observations of the field were performed by the Swift X-ray Telescope, which covered an area of $5 \mathrm{deg}^{2}$ with a sensitivity of $6 \times 10^{-13}-6 \times 10^{-12} \mathrm{erg} \mathrm{cm}^{-2} \mathrm{~s}^{-1}$ (Evans et al. 2016). In this work, we report the serendipitous XMM-Newton (Jansen et al. 2001) slew survey observations of the LIGO localization. In Section 2 we describe our observations, in Section 3 we present our results, and in Section 4 we propose a novel observational strategy in order to maximize the impact of $X M M-N e w t o n$ in the era of GW astronomy.

\section{OBSERVATIONS}

The XMM-Newton Slew Survey is based solely on data from the European Photon Imaging Camera (EPIC) pn camera (Strüder et al. 2001). The in-orbit slew speed of $90^{\circ} \mathrm{hr}^{-1}$ results in an exposure time between $1-11 \mathrm{~s}$. The soft band $(0.2-2 \mathrm{keV})$ sensitivity limit of XMM-Newton slews is $6 \times 10^{-13} \mathrm{erg} \mathrm{cm}^{-2} \mathrm{~s}^{-1}$, close to that of the ROSAT All-Sky Survey (Voges et al. 1999). In the hard $(2-12 \mathrm{keV})$ band the slew data goes significantly deeper $\left(4 \times 10^{-12} \mathrm{erg} \mathrm{cm}^{-2} \mathrm{~s}^{-1}\right)$ than previous surveys. For details on the construction and characteristics of the XMM-Newton slew survey catalog, see Saxton et al. (2008).

At the time of the GW150914 event, XMM-Newton was performing a pointed observation toward the direction of R.A. $=18^{\mathrm{h}} 23^{\mathrm{m}} 14.15$, decl. $=-01^{\circ} 27^{\prime} 38^{\prime \prime} 3$, far from the LIGO localization region of GW150914. Shortly afterwards, 


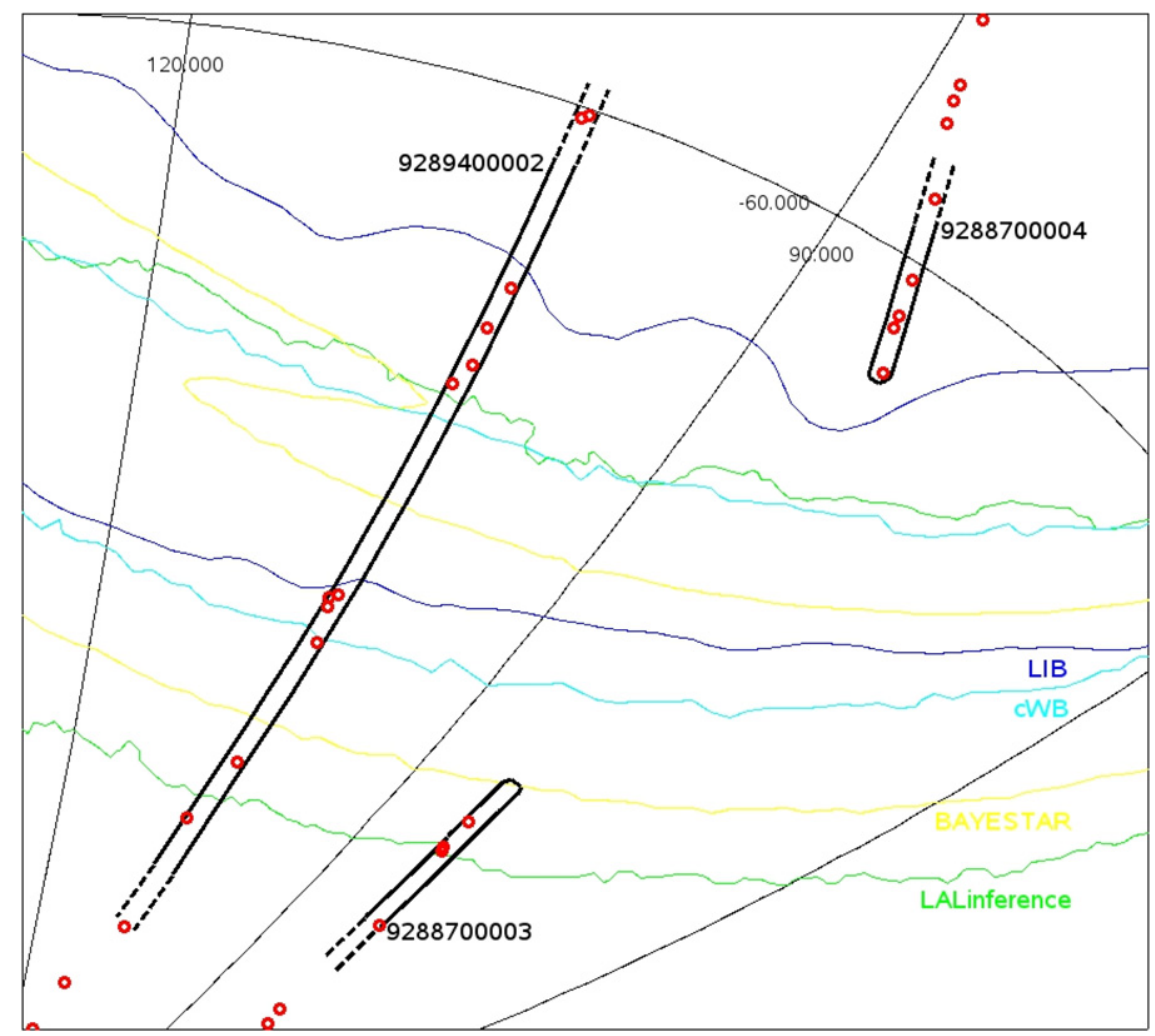

Figure 1. A map of the XMM-Newton slews and slew sources from the two weeks after the GW150914 event. Slew sources are shown as the small circles. Slews are shown as the thin black strips (9288700003 extends to the south, 928870004 to the north, and 9289400002 in both directions). The LIGO $90 \%$ confidence regions from the four different pipelines-LALInference, BAYESTAR, cWB (sky), and LIB—are shown. No other slews in the two week period after the GW150914 event lie anywhere close to the LIGO localization regions.

Table 1

Log of XMM-Newton Slew Observations

\begin{tabular}{lcccc}
\hline \hline OBSID & Start Time & $T-T_{\text {GW }}$ & \multicolumn{2}{c}{ LIGO Coverage } \\
& All $)$ & & $\begin{array}{c}\text { LALInf } \\
\left(\mathrm{deg}^{2}\right)\end{array}$ & $\left(\mathrm{deg}^{2}\right)$ \\
\hline 9288700003 & 2015 Sep 14 11:55:03 & $2.1 \mathrm{hr}$ & 1.1 & 1.1 \\
9288700004 & 2015 Sep 15 01:34:53 & 0.66 day & $\ldots$ & $\ldots$ \\
9289400002 & 2015 Sep 28 23:28:39 & 14.6 day & 6.5 & 4.8 \\
\hline
\end{tabular}

the satellite slewed and by chance crossed the LIGO 90\% error region. Many slews were subsequently made across the sky as the spacecraft moved between its scheduled pointing positions.

Here we analyze the XMM-Newton slews made in the two weeks directly after the GW150914 event. Six slews were made during that time, but only three intercept or come close the LIGO localization maps, as listed in Table 1. The data reduction and analysis used are in many ways similar to the standard slew analysis (Saxton et al. 2008). However, a number of improvements to XMM-Newton slew data analysis have been made, and were used in the present analysis, and these will be described in a future paper (A. M. Read et al. 2016, in preparation).

\section{RESULTS}

A detailed comparison of the slew coverage with the localization regions from the four different LIGO pipelinesLALInference, BAYESTAR, cWB (sky), and LIB - is shown in Figure 1. Since GW150914 is a compact binary coalescence (CBC) event, the LALInference map is considered to be the most accurate, authoritative, and final localization for this event (Abbott et al. 2016e). We therefore prioritize the LALInference map, but still consider all four maps together as a wider total region. We calculate that slew 9288700003 covers $\approx 1.1 \mathrm{deg}^{2}$ of the LALInf (and of the total) region, and slew 9289400002 covers $\approx 4.8 \mathrm{deg}^{2}$ of the LALInf region (and $\approx 6.5 \mathrm{deg}^{2}$ of the total region). Slew 9288700004 marginally overlaps with the LIGO localization and only covers an area outside the $90 \%$ confidence region. These values are tabulated in Table 1.

Source detection was performed using a semi-standard eboxdetect (local) + esplinemap + eboxdetect (map) + emldetect method, and performed on a single image containing the single events $($ pattern $=0$ ) in the $0.2-0.5 \mathrm{keV}$ band, plus single and double events (pattern $=0-4$ ) in the $0.5-12 \mathrm{keV}$ band. Source detection is performed separately in three energy bands: a total band $(0.2-12 \mathrm{keV})$, a hard band $(2-12 \mathrm{keV})$, and a soft band $(0.2-2 \mathrm{keV})$. The source position and background maps are computed separately in each band, and this may result in small differences between the total band and the soft + hard band. The sources detected in the three bands were then combined to produce an initial catalog. Twelve sources were detected in the slew areas coincident with the (total) LIGO region, and X-ray parameters for these are given in Table 2. The sources are also indicated (together with sources detected outside of the LIGO region) in Figure 1. Each detection was assigned a quality flag, from 1 (likely real) to 4 (likely spurious). The detection likelihood (DET_ML) was computed by the emldetect task and is defined as DET_ML $=-\ln P$, where $P$ is the probability the detection is spurious due to a Poissonian fluctuation (Watson et al. 2009). 
Table 2

$X M M-N e w t o n$ Slew Sources Detected within the Total LIGO Localization Region

\begin{tabular}{|c|c|c|c|c|c|c|c|c|c|c|}
\hline Name & $\begin{array}{l}\text { R.A. } \\
\text { (deg) }\end{array}$ & $\begin{array}{l}\text { Decl. } \\
\text { (deg) }\end{array}$ & \multicolumn{3}{|c|}{ Source Counts } & \multicolumn{3}{|c|}{ Detection Likelihood } & Quality & Catalog ID \\
\hline XSS J052742.8-763133 & 81.92836 & -76.52586 & 3.9 & $\cdots$ & 3.9 & 10.5 & $\ldots$ & 10.4 & 2 & 1SWXRT J052742.4-763128 \\
\hline XSS J052914.5-762522 & 82.31060 & -76.42279 & 15.2 & 4.4 & 9.9 & 48.4 & 10.2 & 25.2 & 1 & 1RXS J052918.2-762514 \\
\hline XSS J052952.5-753757 & 82.46876 & -75.63253 & 3.6 & $\cdots$ & $\cdots$ & 8.8 & $\ldots$ & $\cdots$ & 3 & WISE J052952.46-753805.9 \\
\hline XSS J064838.0-641619 & 102.15847 & -64.27199 & 3.7 & $\cdots$ & 3.8 & 9.7 & $\cdots$ & 10.3 & 1 & 1RXS J064837.2-641624 \\
\hline XSS J064903.4-661054 & 102.26428 & -66.18164 & 5.3 & $\ldots$ & 5.5 & 13.0 & $\cdots$ & 15.1 & 2 & WISE J064903.95-661045.2 \\
\hline XSS J064943.8-651636 & 102.43274 & -65.27666 & 3.2 & $\ldots$ & $\cdots$ & 9.0 & $\cdots$ & $\ldots$ & 3 & WISE J064943.68-651638.7 \\
\hline XSS J065106.2-664320 & 102.77593 & -66.72232 & 3.8 & $\ldots$ & $\ldots$ & 11.0 & $\ldots$ & $\ldots$ & 4 & $\cdots$ \\
\hline XSS J065226.0-732221 & 103.10855 & -73.37250 & 3.9 & $\cdots$ & $\cdots$ & 10.1 & $\cdots$ & $\ldots$ & 4 & $\ldots$ \\
\hline
\end{tabular}

Saxton et al. (2008) quote the astrometric uncertainty of slew sources to be about $8^{\prime \prime}$ (68\% confidence error radius), and Warwick et al. (2012) quote a 90\% error circle radius for their slew sample of $10^{\prime \prime}$. Searches for multiwavelength counterparts made use of the facilities at Vizier, Simbad, and NED. Specific cross-correlations were made with WISE and with 2MASS (as in Warwick et al. 2012), and in the case of ROSAT, extra allowances were made to account for the uncertainty in the ROSAT positions. The results are reported in Table 2 (last column). Apart from XSS J065106.2-664320 and XSS J065226.0-732221, the remaining 10 sources all have WISE counterparts within $10^{\prime \prime}$. The chance of a random positional coincidence with a WISE source is only $20 \%$. Among them, four correspond to cataloged X-ray sources. One source, XSS J064838.0-641619, is coincident with the nearby elliptical galaxy NGC 2305 at a distance of $48 \mathrm{Mpc}$. However, it is detected at a flux level consistent with the ROSAT observations. Based on Table 2, we conclude that no new X-ray source was detected in our observation.

\section{STRATEGY}

$X M M$-Newton mainly operates in pointing mode, with a minimum exposure of $5 \mathrm{ks}$ per observation. This timeconstraint makes it highly impractical to rapidly tile large areas of the sky or to follow-up a large number of candidates. Slew observations are executed only between two pointed observations. Indeed, the $90 \%$ error region of GW150914 was partially covered by XMM-Newton slews only by chance. Nevertheless, the observations presented in the previous section covered $6 \mathrm{deg}^{2}\left(8 \mathrm{deg}^{2}\right)$ of the LALInf (total) credible region with a sensitivity comparable to the Swift/XRT follow-up (Evans et al. 2016). Here we explore the possibility of performing XMM-Newton Target of Opportunity Slew Surveys in response to future $\mathrm{GW}$ triggers.

Given the $14^{\prime}$ radius EPIC-pn field of view and the slew speed of $90^{\circ} \mathrm{hr}^{-1}$, the $590 \mathrm{deg}^{2}$ area of the GW150914 error region could in principle be covered in about $14 \mathrm{hr}$. However, overhead times could significantly increase the total time required to perform such a large area survey. Their impact can be evaluated on the basis of a special test for a large area slew survey that was performed at a reduced speed in 2006 September (revolution 1242). This slow slew survey covered a $140 \mathrm{deg}^{2}$ sky region (a rectangle of $40^{\circ} \times 3.5$; see Figure 2 ) within a single satellite orbit ( $\sim 1.5$ days of scientific observing time) with 16 partially overlapping slews at a reduced speed of $30^{\circ} \mathrm{hr}^{-1}$. During this test, the time overhead between two adjacent slews was $\sim 3 \mathrm{ks}$. A similar survey at the normal slew speed of $90^{\circ} \mathrm{hr}^{-1}$ would take only $\sim 20 \mathrm{hr}$ and an area larger than $40 \%$ of the GW150914 error region could be covered within a single XMM-Newton orbit. Without requiring an overlap of the slews, the area surveyed in an orbit would increase to more than $70 \%$ of the GW150914 error region. Furthermore, in future runs the localization accuracy of GW transients is expected to improve thanks to the increased sensitivity, larger bandwidth and the addition of other detectors to the GW network. A 50\% reduction in the error region is already expected for the $\mathrm{O} 2$ run (2016-2017), with $14 \%$ of the detections being localized within $20 \mathrm{deg}^{2}$ (Abbott et al. 2016d), an area which could be covered by slews in $\approx 30 \mathrm{ks}$. By 2019 this fraction is expected to increase up to $\approx 30 \%$ (Abbott et al. 2016d).

The actual observing efficiency also depends on the sky position of the GW trigger, which determines the slew length, its direction, and the sky regions that cannot be observed due to the satellite visibility constraints. About $1 / 6$ of the sky is accessible by XMM-Newton at any given time, and some slewing directions may require more complex satellite operations and produce a worse astrometric accuracy, possibly causing an imperfect coverage of the region to be surveyed. Nonetheless, even if the whole error region cannot be covered due to visibility and slewing constraints, the proposed strategy will be an efficient way to search a significant fraction of a large sky area for an X-ray counterpart.

The typical XMM-Newton response time to a ToO trigger is $\lesssim 10-12 \mathrm{hr}$, and in the best case, it can be as small as 4 to $5 \mathrm{hr}$ (e.g., Schartel 2015). As we discuss later on, a very fast turnaround would be preferable in some exceptional cases (e.g., a joint GRB-GW detection or a well-localized GW source), and a slower reaction time is instead preferred to search for off-axis afterglow emission (Granot et al. 2002; van Eerten \& 


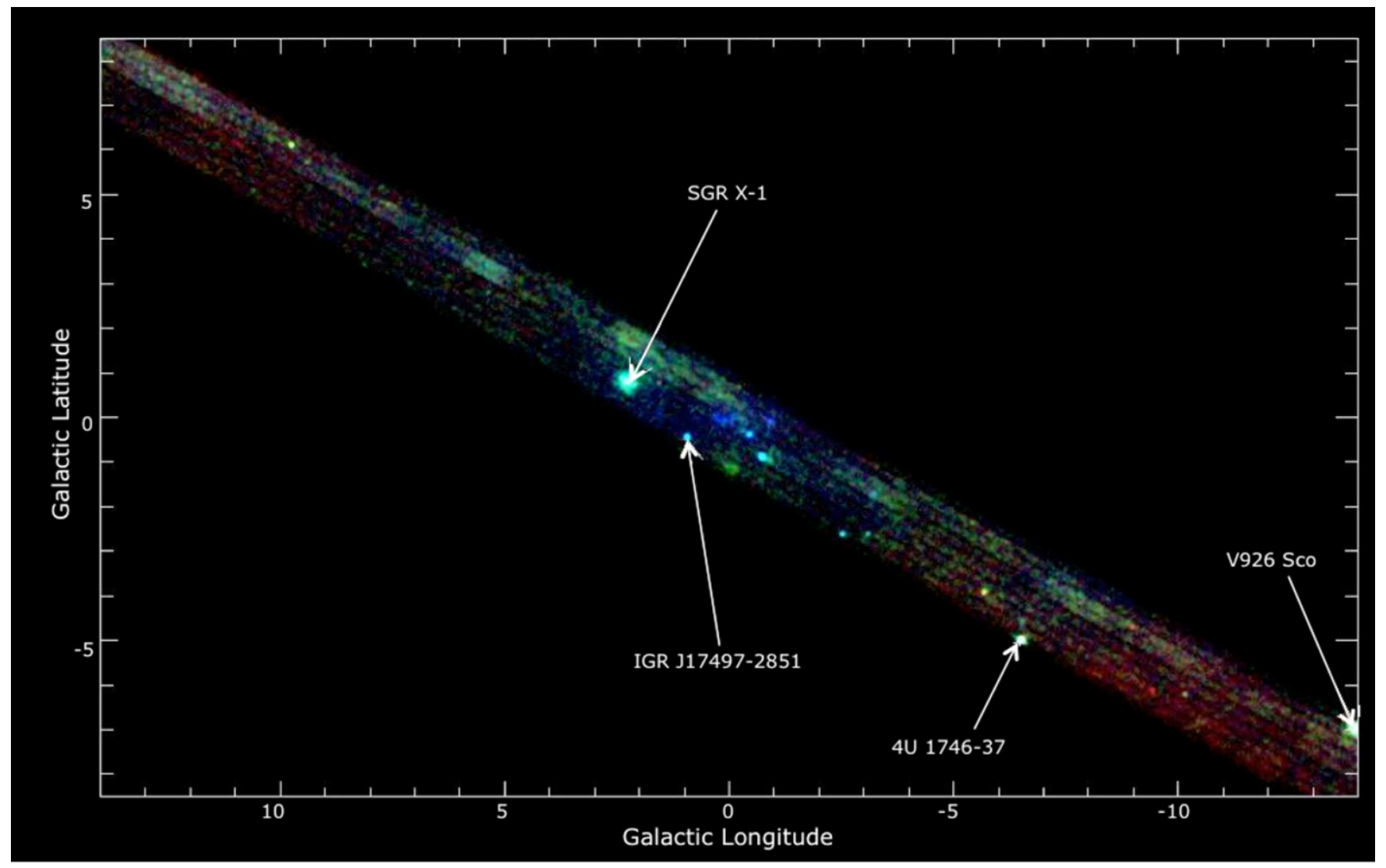

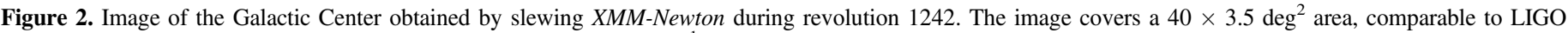

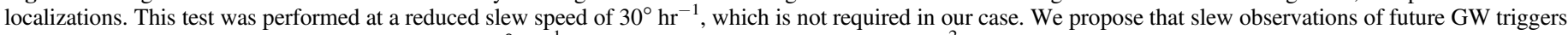
should be performed at a standard slew speed of $90^{\circ} \mathrm{hr}^{-1}$. This would allow us to cover a $20 \mathrm{deg}^{2}$ area in $\approx 30 \mathrm{ks}$.

MacFadyen 2011). In order to maximize the scientific return, the data should be analyzed in "real time" to identify and localize possible candidates with sufficient accuracy to enable further follow-up campaigns. At the moment a fast automatic analysis is performed on slew data, but it requires orbital data that become available with a delay of 1-2 weeks. Without the orbital data, the slew source positions within a few arcmin could be obtained directly from raw slew data, relying on the nominal slewing direction and the relative position of known bright X-ray sources. This would make it possible to reduce the processing time to few hours.

In Figure 3 we estimate the detectability of short GRB afterglows during an XMM-Newton slew observation. First, we considered the known sample of Swift short GRBs with measured redshift (e.g., D'Avanzo et al. 2014). The left panel shows their X-ray fluxes distribution at $t=1$ day and at a distance $D=200 \mathrm{Mpc}$, which is the horizon distance for NSNS mergers at the LIGO design sensitivity. Most of them $(>60 \%)$ lie above the XMM-Newton slew survey detection threshold, $f_{X}=1.2 \times 10^{-12} \mathrm{erg} \mathrm{cm}^{-2} \mathrm{~s}^{-1}(0.2-12 \mathrm{keV})$. However, one has to consider that the observed sample is shaped by complex observational biases, which tend to favor the detection and localization of the brightest events. We therefore simulated a sample of GRB afterglows following standard prescriptions for a spherical fireball (Granot \& Sari 2002), and post-jet-break scalings from Sari et al. (1999). The explosion properties of short GRBs are not well constrained, and we made an educated guess about the values and distributions of afterglow parameters. We assumed an electron spectral index $p \approx 2.3$, and simulated three populations of explosions with the following parameters.

a. A total energy release $E$ uniformly distributed between $10^{48}$ and $10^{51} \mathrm{erg}$, a medium density $n$ between 0.001 and $1 \mathrm{~cm}^{-3}$, an electron energy fraction $\epsilon_{e}$ between $10^{-3}$ and 0.1 , a magnetic energy fraction $\epsilon_{B}$ between $10^{-4}$ and $10^{-2}$, and an opening angle $\theta_{j}$ between $1^{\circ}$ and $40^{\circ}$.

b. A total energy release with an average $\langle E\rangle=10^{49} \mathrm{erg}$ and Gaussian dispersion $\sigma_{E}=1 \mathrm{dex}, n$ between 0.001 and $1 \mathrm{~cm}^{-3},\left\langle\epsilon_{e}\right\rangle=0.1$ with Gaussian dispersion $\sigma_{e}=0.5 \mathrm{dex}$, $\epsilon_{B}$ between $10^{-4}$ and $10^{-2}$, and $\left\langle\theta_{j}\right\rangle=10^{\circ}$ with Gaussian dispersion $\sigma_{\theta}=0.5$ dex.

c. $\langle E\rangle=10^{50} \mathrm{erg}$ with Gaussian dispersion $\sigma_{E}=1 \mathrm{dex}$, $\langle n\rangle=0.1$ with $\sigma_{n}=1 \mathrm{dex},\left\langle\epsilon_{e}\right\rangle=0.1$ with $\sigma_{e}=0.5 \mathrm{dex}$, $\left\langle\epsilon_{B}\right\rangle=0.1$ with $\sigma_{B}=0.5 \mathrm{dex}$, and $\left\langle\theta_{j}\right\rangle=10^{\circ}$ with $\sigma_{\theta}=0.5$ dex.

For each set of parameters, we simulated 340,000 afterglows and calculated their X-ray fluxes in the $0.2-12 \mathrm{keV}$ at $t=1$ day. We assumed an intrinsic absorption $N_{H}$, ${ }_{i}=10^{21} \mathrm{~cm}^{-2}$, and a flat galactic column density $N_{H}$, $g=3 \times 10^{20} \mathrm{~cm}^{-2}$. The results are shown in Figure 3 (middle panel), where we report the cumulative flux distribution. For the most optimistic case (c) the fraction of visible X-ray afterglows is nearly $80 \%$, and for the less optimistic scenario (a) is still $25 \%$. These estimates should be taken with a grain of salt, as they simplify a complex and poorly constrained phenomenon. The predicted fluxes, calculated for on-axis observers, are significantly fainter if the jet is seen far off-axis 

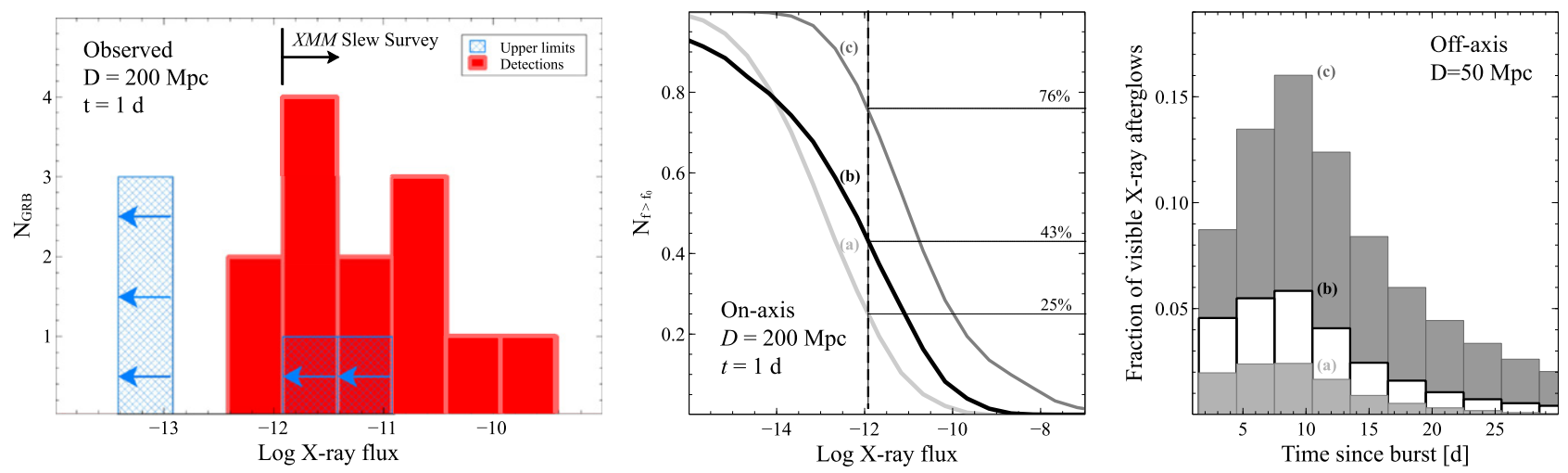

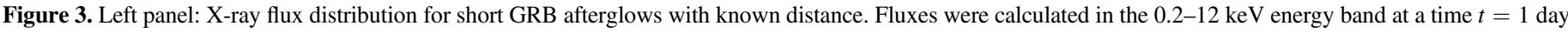

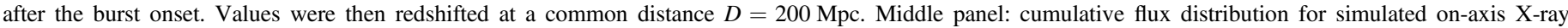

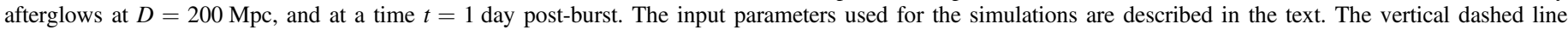

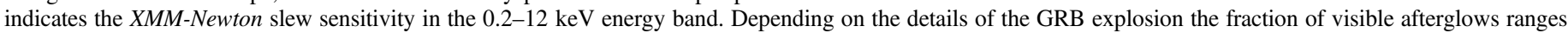

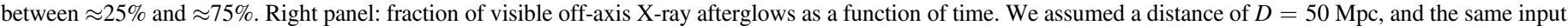
parameters of on-axis simulations. The peak ranges between $\approx 3 \%$ and $\approx 15 \%$ at $t \approx 8$ days post-burst.

$\left(\theta_{\text {obs }}>\theta_{j}\right)$. Viewing angle effects could therefore affect our calculations, decreasing the number of visible X-ray afterglows by a factor $\sim 3$ to over 100 , depending on the (unknown) beaming distribution of short GRB jets. This may be alleviated by the fact that the source inclination impacts not only the afterglow flux, but also the strength of the emitted gravitational radiation. Face-on binaries, i.e., with their rotation axis pointing toward us, are more likely to be detected by a factor of 3.4 (Schutz 2011). It is plausible that the GRB jet will form along the same axis, thus suggesting that on-axis short GRBs should also be stronger GW sources. A joint GW-GRB detection would give us a high level of confidence to search for an on-axis X-ray afterglow. However, even in this case, a standard follow-up strategy could not be effective. Given its large field of view, the Fermi Gamma-ray Burst Monitor is most likely to detect such a joint event, thus providing only a coarse localization (e.g., Connaughton et al. 2016) comparable to the LIGO one.

In the absence of a GRB trigger, the most likely counterpart would be an orphan afterglow, whose emission is much fainter, and therefore detectable within a much smaller volume. By adopting the simple analytical model of Granot et al. (2002) with the same explosion parameters listed above, and folding in the probability distribution of Schutz (2011) for the binary orientation, we derive in Figure 3 (right panel) the prospects for detecting such off-axis emission with XMM-Newton. For a distance of $50 \mathrm{Mpc}$, the fraction of detectable events ranges between $3 \%$ and $15 \%$, much smaller than for an on-axis explosion. However, Figure 3 also shows that the optimal reaction time is slow, peaking between 7 and 15 days after the burst.

Finally, we consider that the past decade of afterglow studies has unveiled the presence of new emission components in addition to the standard forward shock emission described in Granot \& Sari (2002). Of particular interest is the emission from a stable millisecond magnetar, which could power a bright and nearly isotropic X-ray transient. Zhang (2013) estimated a bright and persistent $\mathrm{X}$-ray emission from a magnetar-driven relativistic wind lasting for several hours after the burst onset. Metzger \& Piro (2014) instead considered the emission from the remnant pulsar wind nebula, and estimated a peak X-ray luminosity of $10^{43}-10^{44} \mathrm{erg} \mathrm{s}^{-1}$ on a timescale of $\lesssim 1$ day. At a distance of $200 \mathrm{Mpc}$, this corresponds to a flux of $2 \times 10^{-12}-2 \times 10^{-11} \mathrm{erg} \mathrm{cm}^{-2} \mathrm{~s}^{-1}$, above the XMM-Newton slew survey sensitivity. In case of a particularly well-localized GW event $\left(\approx 20 \mathrm{deg}^{2}\right)$, we argue that a rapid observation of the GW field would still be extremely valuable in order to search for these possible X-ray counterparts. Within this field we expect to detect on average $10 \mathrm{X}$-ray candidates with high significance, which can be easily targeted for subsequent follow-up with Swift or optical/nIR ground-based facilities.

\section{SUMMARY}

We report XMM-Newton observations of the field of GW150914, the first LIGO detection. Although no X-ray counterpart was found, these serendipitous observations show the great potential of XMM-Newton slews for searching for electromagnetic counterparts of GWs. A single slew of only 7 minutes covered $4.8 \mathrm{deg}^{2}$ of the LIGO region down to a sensitivity of $6 \times 10^{-13} \mathrm{erg} \mathrm{cm}^{-2} \mathrm{~s}^{-1}(0.2-2 \mathrm{keV})$. An observing strategy consisting of a series of adjacent slews (Figure 2) could survey a large fraction of future LIGO localizations within a single $X M M$-Newton orbit. In order to maximize the chances of success without excessively impacting XMMNewton operations, we suggest three possible responses to future GW triggers: (a) a rapid ( $\lesssim 1$ day) high-priority ToO of the $\mathrm{GW}$ region in case of a simultaneous GRB trigger with poor localization; (b) a slower response ( $\approx 7$ days) ToO in case of a rare, nearby ( $\lesssim 50 \mathrm{Mpc}) \mathrm{GW}$ transient; and (c) a rapid ToO in case of a well-localized $\left(\approx 20 \mathrm{deg}^{2}\right) \mathrm{GW}$ event. The latter case could be assigned a medium-priority status, i.e., be implemented only during working hours. On the basis of the experience of the XMM-Newton Slew Survey, we expect about 0.5 candidates per square degree, much less than the sources expected from deep optical surveys. Multi-wavelength followup observations may be required in order to characterize and identify the selected sources. For a canonical fireball spectral index $\beta_{\mathrm{OX}} \gtrsim 0.6$, the optical counterparts of XMM-Newton slew candidates are expected to have $r \lesssim 22 \mathrm{mag}$, which can be reached with small aperture $(\sim 1.5 \mathrm{~m})$ telescopes in reasonably short $(\lesssim 10$ minute $)$ exposures.

We thank Richard Saxton and the anonymous referee for useful and constructive comments that helped improve the manuscript. This work is based on observations obtained with $X M M-N e w t o n$, an ESA science mission with instruments and 
contributions directly funded by ESA Member States and NASA. This research has made use of the SIMBAD database, operated at CDS, Strasbourg, France, and of the NASA/IPAC Extragalactic Database (NED), which is operated by the Jet Propulsion Laboratory, California Institute of Technology, under contract with the National Aeronautics and Space Administration.

\section{REFERENCES}

Aasi, J., Abbott, B. P., Abbott, T., et al. 2015, CQGra, 32, 074001 Abbott, B. P., Abbott, R., Abbott, T. D., et al. 2016a, ApJL, 818, L22

Abbott, B. P., Abbott, R., Abbott, T. D., et al. 2016b, PhRvL, 116, 061102

Abbott, B. P., Abbott, R., Abbott, T. D., et al. 2016c, arXiv:1602.03843

Abbott, B. P., Abbott, R., Abbott, T. D., et al. 2016d, LRR, 19, 1

Abbott, B. P., Abbott, R., Abbott, T. D., et al. 2016e, ApJL, submitted (arXiv:1602.08492)

Abbott, B. P., Abbott, R., Abbott, T. D., et al. 2016f, arXiv:1602.03841

Belczynski, K., Dominik, M., Bulik, T., et al. 2010, ApJL, 715, L138

Connaughton, V., Burns, E., Goldstein, A., et al. 2016, arXiv:1602.03920
D’Avanzo, P., Salvaterra, R., Bernardini, M. G., et al. 2014, MNRAS, 442, 2342

Evans, P. A., Kennea, J. A., Barthelmy, S. D., et al. 2016, MNRAS, in press (arXiv:1602.03868)

Granot, J., Panaitescu, A., Kumar, P., \& Woosley, S. E. 2002, ApJL, 570, L61 Granot, J., \& Sari, R. 2002, ApJ, 568, 820

Jansen, F., Lumb, D., Altieri, B., et al. 2001, A\&A, 365, L1

Kann, D. A., Klose, S., Zhang, B., et al. 2011, ApJ, 734, 96

Metzger, B. D., \& Piro, A. L. 2014, MNRAS, 439, 3916

Murase, K., Kashiyama, K., Meszaros, P., Shoemaker, I., \& Senno, N. 2016 ApJL, in press (arXiv:1602.06938)

Perna, R., Lazzati, D., \& Giacomazzo, B. 2016, ApJL, 821, L18

Sari, R., Piran, T., \& Halpern, J. P. 1999, ApJL, 519, L17

Savchenko, V., Ferrigno, C., Mereghetti, S., et al. 2016, ApJL, 820, L36

Saxton, R. D., Read, A. M., Esquej, P., et al. 2008, A\&A, 480, 611

Schartel, N. 2015, GCN, 18154, 1

Schutz, B. F. 2011, CQGra, 28, 125023

Strüder, L., Briel, U., Dennerl, K., et al. 2001, A\&A, 365, L18

van Eerten, H. J., \& MacFadyen, A. I. 2011, ApJL, 733, L37

Voges, W., Aschenbach, B., Boller, T., et al. 1999, A\&A, 349, 389

Warwick, R. S., Saxton, R. D., \& Read, A. M. 2012, A\&A, 548, A99

Watson, M. G., Schröder, A. C., Fyfe, D., et al. 2009, A\&A, 493, 339

Zhang, B. 2013, ApJL, 763, L22 